\title{
Magnetic nanoparticles for medical applications
}

First draft submitted: 27 January 2017; Accepted for publication: 7 February 2017; Published online: 24 March 2017

Magnetic targeting of endothelial cells to stented arteries

Evaluation of: Polyak B, Medved

M, Lazareva $\mathrm{N}$ et al. Magnetic

nanoparticle-mediated targeting

of cell therapy reduces in-stent stenosis in injured arteries. ACS Nano

10, 9559-9569 (2016). doi:10.1021/

acsnano.6b04912

Myocardial ischemia due to coronary artery stenosis or atherosclerotic plaque rupture is commonly treated by percutaneous intervention with stent angioplasty. In the USA alone, more than 2 million patients per year undergo vascular stent implantation [1]. However, the stent implantation procedure leads to the disruption of endothelial monolayer and to an excessive smooth muscle cell proliferation, which in the longer term can cause restenosis. Drug-eluting stents decrease hyperplastic responses, leading to reduced restenosis as compared with bare metal stents, but in parallel with inhibition of hyperplasia, they also prevent re-endothelialization of the injured artery.

In their paper, Polyak et al. utilized magnetic targeting of endothelial cells (ECs) as a therapeutic strategy to promote local repair of the injured vascular intima in a rat carotid artery stenting model. For this purpose, rat aortic ECs were isolated and loaded with biodegradable polylactide-based particles containing nanocrystalline magnetite (magnetic nanoparticles [MNPs]). The MNPs had a hydrodynamic diameter of $278 \mathrm{~nm}$, a $\zeta$-potential of $-14.4 \mathrm{mV}$, and their magnetization measured at $0.5 \mathrm{~T}$ was $24.6 \mathrm{emu} / \mathrm{g}$ of composite [2].

In the biodistribution studies using MNPs labeled with a lipophilic near-infrared (NIR) fluorescent probe, the authors demonstrated that intravenous administration leads to the capture of rigid, MNP-loaded cells in the lungs. As only an extremely small number of viable cells can pass lung capillaries, magnetic targeting of ECs administered via tail vein to a stent implanted in carotid artery was unsucauthors investigated the efficacy of magnetic targeting upon local delivery of MNP-loaded ECs and the long-term therapeutic outcomes.

ECs were administered at the distal end of the stented artery under stop-flow conditions and targeted to the stent using an external magnetic field of $0.14 \mathrm{~T}$ generated across the neck area for $12 \mathrm{~min}$. At 1 week postdelivery, bioluminescent imaging detected about 30\% of administered cells in the stented artery segment, whereby a higher signal density was observable in the distal stent region, closer to the delivery catheter.

The effects of therapeutic cell delivery were monitored over 2 months using ultrasound imaging to assess the lumen diameter and hemodynamic changes in the artery, and were subsequently confirmed by postmortem histology. Magnetic targeting of ECs caused a 1.7-fold less reduction in lumen diameter at the distal part of the stent as compared with both the respective proximal stent region and the control group (stented, untreated), and 2.3-fold less reduction in peak systolic velocities. Moreover, a significant, 2.1-fold reduction of vessel stenosis relative of control group was observed at the distal stent region by histology, consistent with the iron oxide nanoparticle localization detected with Prussian blue staining.

Taken together, despite some limitations concerning the uniformity of cell delivery, cessful. In the subsequent experiments, the
Iwona Cicha', Stefan Lyer ${ }^{1}$, Christina Janko', Ralf P Friedrich ${ }^{1}$, Marina Pöttler ${ }^{1}$ \& Christoph Alexiou ${ }^{*, 1}$

'Department of Otorhinolaryngology, Head \& Neck Surgery, Section of Experimental Oncology \& Nanomedicine (SEON), Else Kröner-Fresenius-StiftungProfessorship, Universitätsklinikum Erlangen, Friedrich-Alexander-Universität Erlangen-Nürnberg, Glueckstr. 10a, 91054 Erlangen, Germany

*Author for correspondence: c.alexiou@web.de 
cell source and stent material, the promotion of local endothelial healing by magnetic cell delivery seems to be a promising approach in order to prevent in-stent restenosis and thrombosis.

- I Cicha

\section{siRNA delivery with novel magnetic core-shell nanoparticles}

Evaluation of: Xiong L, Bi J, Tang Y, Qiao S. Magnetic core-shell silica nanoparticles with large radial mesopores for siRNA delivery. Small 12(34), 4735-4742 (2016).

To achieve any considerable improvement in the treatment of diseases, timely delivery of the drug to the target tissue is necessary. In particular, the delivery of siRNAs for gene therapy is a big challenge due to the risk of their rapid degeneration by enzymes [3], but also due to their negative charge, which dramatically limits their cellular uptake.

Using magnetic mesoporous silica nanoparticles for siRNA drug delivery has been proposed to solve these problems. However, mesoporous silica nanoparticles are often bigger than $200 \mathrm{~nm}$, which limits an effective cellular uptake and usually leads to a rapid clearance by the liver in vivo. The large volume fraction of the silica may also result in a relatively low magnetic responsiveness of such composites. Furthermore, achieving a proper relation between siRNA loading capacity that increases with the pore size and volume, and controlled release is difficult.

In their manuscript, Xiong et al. described the development of a new nanoparticle system with an iron oxide core and a mesoporous silica shell, which they proposed as a platform for siRNA-delivery. The authors used sophisticated methods for analyzing each synthesis step of the nanosystem, which in the end is additionally coated by a tannic acid layer to improve an effective and controlled drug release in the targeted cells. The methods, ranging from different electron microscopies via energy dispersive $\mathrm{x}$-ray spectrum, $\mathrm{x}$-ray diffraction to Raman spectroscopy, showed the detailed physicochemical properties of each interstage. Each step of the synthesis was analyzed in a logical and comprehensible way, leading to a stable nanosystem of $150 \mathrm{~nm}$ hydrodynamic diameter with mesopores of $12 \mathrm{~nm}$ and a pore volume of $1.13 \mathrm{~cm}^{3} / \mathrm{g}$. Based on the analyses of the properties, the loading capacity of $2 \mathrm{wt} \%$ seemed to be optimal for siRNA delivery, and the effective protection of the siRNAs after the final coating with tannic acid was confirmed. In the subsequent in vitro investigations, the authors demonstrated a good biocompatibility of all relevant interstage particles, as well as that of the final system loaded with an anti-EGFP-siRNA up to concentrations of $200 \mu \mathrm{g} /$ $\mathrm{ml}$. On the other hand, the particles reduced viability of the osteosarcoma cell line KHOS by $50 \%$, if they were loaded with siRNAs directed against the oncogene PLK1. This toxic effect on cancer cells was further enhanced using an external magnetic field.

Taken together, Xiong et al. demonstrated the excellent physicochemical properties and in vitro efficacy of a newly designed magnetic composite nanosystem for siRNA delivery. A potential source of concern with regard to human application could be the use of $\mathrm{Al}^{3+}$ as an excipient for the tannic acid coating, since aluminum is known to have unfavorable effects on living organisms [4]. Nonetheless, it will be interesting to follow further studies on this nanosystem, in particular, its efficacy in vivo in respect to drug delivery and tumor therapy.

- S Lyer

\section{Magnetic particles catalyzing antibacterial drug release at infection sites}

Evaluation of: Ji H, Dong K, Yan Z et al. Bacterial hyaluronidase self-triggered prodrug release for chemo-photothermal synergistic treatment of bacterial infection. Small 12(45), 6200-6206 (2016).

Overuse of antibiotics led to development of bacterial resistances, which reduce therapeutic efficacy and increase mortality [5]. There is an urgent need for alternative therapeutic strategies to fight bacterial infections. Ji et al. present a nanoparticle-based approach to generate hydroxyl radicals $(\bullet \mathrm{OH})$ with high antibacterial activity. Ascorbic acid (AA) can act as a prodrug for $\mathrm{H}_{2} \mathrm{O}_{2}$, which itself slowly generates antibacterial $\bullet \mathrm{OH}$, and in the presence of peroxidase the conversion is accelerated. Ferromagnetic nanoparticles (MNPs, $\left.\mathrm{Fe}_{3} \mathrm{O}_{4}\right)$ possess a peroxidase-like activity and serve as a catalyst to transform AA into $\bullet \mathrm{OH}$ [6]. Combining these features, an 'on-demand' drug delivery platform was constructed by loading the nontoxic AA into the pores of graphene-mesoporous silica nanosheets (GS) followed by their encapsulation within hyaluronic acid-dopamine conjugates (HA-DA). Finally, the MNPs were bound to the outer surface, resulting in AA@GS@HA-MNPs. By precoating the MNPs with vancomycin, which targets gram-positive and gramnegative bacteria, the nanocarrier can be concentrated at infection sites where hyaluronidase is generated by bacteria, degrading the HA-DA. Subsequently, AA is released and converted into $\bullet \mathrm{OH}$ by MNPs. Due to NIR light absorption properties of graphene, synergistic photothermal therapy can be employed [7].

Since optimum of the peroxidase-like activity of GS@HA-MNPs was at $\mathrm{pH} 4.0$ and $30^{\circ} \mathrm{C}$, generation of $\bullet \mathrm{OH}$ from AA in the presence of GS@HA-MNPs 
should be efficient at infection sites with low $\mathrm{pH}$ values $(\sim \mathrm{pH} 5.5)$ due to anaerobic fermentation and inflammation. Indeed, incubation of GS@HA-DA with hyaluronidase or bacteria resulted in efficient degradation of the HA-DA and release of encapsulated AA. GS@ HA-MNPs reduced survival of Escherichia coli and Staphylococcus aureus only in the presence of AA, proofing the catalyst function of GS@HA-MNPs. Furthermore, GS@HA-MNPs with encapsulated AA (AA@ GS@HA-MNPs) showed even higher antibacterial activity compared with free AA, possibly because of the specific targeting to the bacterial surface and the in situ formation of AA. Transmission electron microscopy and scanning electron microscopy images documented the adherence of MNPs on bacterial membranes and their disruption due to lipid oxidation by $\bullet \mathrm{OH}$ after treatment with AA@GS@HA-MNPs. Importantly, GS@HAMNPs with encapsulated AA showed a negligible toxicity against mammalian HEK293T cells compared with that against bacteria. Also, S. aureus within biofilms were efficiently killed byAA@GS@HA-MNPs; existing biofilms were eliminated and new biofilms inhibited as well. Evaluating the effects of AA@GS@HA-MNPs in vivo, the implant-related periprosthetic infection model was employed, in which medical catheters with $S$. aureus biofilms were subcutaneously implanted into mice. Whereas control mice showed swellings and purulence at the infection site, mice treated with AA@GS@HAMNPs and synergistic NIR irradiation showed gradual, or almost completed, healing of the wounds, respectively. Histology revealed bacteria, tissue lesions and cell debris in the untreated control group, whereas no signs of infections were found in the synergistic treatment group.

In conclusion, a functional platform for the delivery of the prodrug AA to treat bacterial infections was generated using the bacterial hyaluronidase-sensitive GS@ HA-MNPs as nanocarriers. These particles represent a promising strategy to overcome persistent bacterial infections.

- C Janko \& C Alexiou

\section{Intracellular delivery of multifunctional nanoparticles for controlled axon formation during neuronal development}

Evaluation of: Suarato G, Lee SI, Li W et al. Micellar nanocomplexes for biomagnetic delivery of intracellular proteins to dictate axon formation during neuronal development. Biomaterials 112, 176-191 (2017).

Cell polarity, a feature of many cell types, often determines their specific functions. During embryonic development, polarization ascertains the precise dorsal-ventral, left-right and superior-inferior axis of the body and governs cell migration by asymmetric cell divi- sion through highly regulated concentration gradients of proteins in the cells or across the body [8]. Notable examples of polarized cells are epithelial cells with apical-basal polarity and neurons with functionally and morphologically distinct compartments of dendrite and axon that allow the directional flow of signals in the brain. Disturbed subcellular localization of specific proteins may lead to cancer, dysplasia and neuropathologies $[9,10]$. Thus, the ability to control the spatiotemporal protein localization could enable new therapies for the diseases caused by disturbed cell polarity.

In their recent work, Suarato et al. took the initial steps toward developing this technology. They aim was to manipulate axon formation by directed subcellular introduction and retention of LKB1, an intracellular kinase crucial for axon development [11]. For that purpose, two different self-assembled, LKB1-functionalized and hydrophobically modified glycol chitosan (HGC) nanocomplexes were produced to ensure subcellular delivery of LKB1 to developing neurons. To enable tracking of the nanosystems, Cy5.5-LKB1HGC-NC were labeled with Cy5.5 fluorophores, and MNP-LKB1-HGC-NC were complexed with fluorescent superparamagnetic iron oxide nanoparticles (MNP). The usage of chitosan enabled a rapid cellular uptake into the cytoplasm, circumventing endosomal entrapment and lysosomal degradation [12].

In vitro, neurons incubated with LKB1 nanocomplexes showed prolonged cytoplasmic stability and availability of LKB-1, without negative effects on viability. Biochemical analysis demonstrated that nanocomplexed LKB1 retained its kinase activity and phosphorylated SAD-A kinases, which in turn phosphorylate the microtubule-associated protein $\mathrm{T}$, involved in the growth, differentiation and stabilization of axons, and required for LKB1-mediated axon formation [13]. Additionally, co-immunostainings of cultured hippocampal neurons with axonal and neuronal markers revealed that LKB1 nanocomplexes induced the formation of multiple axons in approximately $25 \%$ of cells, albeit with no effect on the number of dendrites, whereas in control cultures, less than $10 \%$ of neurons formed more than one axon.

Finally, the authors established a methodology of localized intracellular micelle delivery into live rodent embryonic brains in utero. After C-section of E18-time-pregnant rats and exposure of the uterine horns, fluorescently labeled chitosan micelles were injected through the uterine membrane into one of the lateral ventricles of the living embryonic brain. After continuing the embryonic development for 2 days, the cortical brain slices were obtained at E20. High-resolution images showed uptake of chitosan micelles in cortical progenitor cells at the cortical plate, or radially 
migrating cells, without affecting neuronal survival or development. Future work is intended to evaluate if LKB-1 nanocomplexes containing MNPs enable localized subcellular accumulation of the protein and the magnetic control of directional axon formation.

\section{- RP Friedrich}

\section{Magnetic ranking of circulating tumor cell phenotypes}

Evaluation of: Poudineh M, Aldridge PM, Ahmed $\mathrm{S}$ et al. Tracking the dynamics of circulating tumour cell phenotypes using nanoparticle-mediated magnetic ranking. Nat Nanotechnol. doi: 10.1038/nnano.2016.239 (2016) (Epub ahead of print).

Metastatic disease is responsible for $90 \%$ of cancerrelated deaths. Because circulating tumor cells (CTCs) are a source of metastasis, their comprehensive characterization is expected to improve cancer diagnosis and therapy. Poudineh et al. describe a novel approach to classify CTCs, termed magnetic ranking cytometry (MagRC), based on nanoparticle-mediated cell sorting in a chip architecture, which can be controlled via a magnetic field applied along a channel. Using information assembled at the single-cell level, MagRC generates a phenotypic profile, which permits sorting of CTCs into 100 capture zones that allow us to diversify the tracked CTCs as a function of tumor growth and aggressiveness. Moreover, this system is able to profile CTCs even at levels of 10 cells $/ \mathrm{ml}$ in unprocessed blood. Whole blood is incubated with antibody-functionalized magnetic nanoparticles which bind specifically to a corresponding surface marker expressed on cells of interest. Microengineered $\mathrm{x}$-shaped structures within the channel support the profiling capacity of MagRC by generating regions of slow flow, where cells tagged with magnetic nanoparticles can be captured with the magnetic field even with very low levels of magnetic loading. High magnetic field gradients are achieved via different-sized nickel micromagnets positioned concentrically within $\mathrm{x}$-shaped structures, whereby sorting of cells with high or low marker expression can be accomplished. Combined with immunostaining of captured cells from whole blood, this system enables high-resolution CTC profiling.

\section{References}

1 Kim LK, Feldman DN, Swaminathan RV et al. Rate of percutaneous coronary intervention for the management of acute coronary syndromes and stable coronary artery disease in the United States (2007 to 2011). Am. J. Cardiol. 114, 1003-1010 (2014).

2 Adams CF, Rai A, Sneddon G, Yiu HH, Polyak B, Chari DM. Increasing magnetite contents of polymeric magnetic
As a proof of concept, the authors monitored the growth of human breast cancer, induced in immunodeficient mice, with or without pretreatment with estrogens. Blood samples collected every 10 days were analyzed with MagRC using magnetic nanoparticles coated with epithelial cell adhesion molecule (CAM) antibody. The more aggressive, estrogen-stimulated tumor group exhibited much higher CTC numbers as the study progressed, concomitant with a phenotypic shift and micrometastases in the lung. Unstimulated mice had stable CTC profiles and remained micrometastasis free. The occurrence of the metastases in parallel with the changes in CTC profile analyzed by MagRC was consistent with the hypothesis that the CTCs originating from the estrogen-stimulated tumors have a more invasive profile.

In subsequent analyses, MagRC was performed in blood samples from patients with metastatic castrationresistant prostate cancer, patients with localized prostate cancer and healthy donors. The variety of CTC profiles within the metastatic castration-resistant prostate cancer group was low, consistent with low epithelial CAM production. In patients with localized prostate cancer, CTCs were more diverse and were profiled along with the Gleason score. CTCs captured in earlier zones of MagRC were scored as G6 and those in later zones were from G8 and G9 tumors, suggesting that MagRC can help clarifying the differences between tumors with similar staging data.

Taken together, the advantages of MagRC include the usage of unprocessed blood samples, a higher sensitivity at lower cell number and the ability to report a protein expression profile. This technique can also be adapted to a variety of diseases where diagnostics is based on antibodies and can be used in clinical cancer management with further effort at system integration.

- M Pöttler

Financial \& competing interests disclosure

The authors have no relevant affiliations or financial involvement with any organization or entity with a financial interest in or financial conflict with the subject matter or materials discussed in the manuscript. This includes employment, consultancies, honoraria, stock ownership or options, expert testimony, grants or patents received or pending, or royalties.

No writing assistance was utilized in the production of this manuscript.

particles dramatically improves labeling of neural stem cell transplant populations. Nanomedicine 11, 19-29 (2015).

3 Tekade RK, Tekade M, Kesharwani P, D'Emanuele A. RNAi-combined nano-chemotherapeutics to tackle resistant tumors. Drug Discov. Today 21, 1761-1774 (2016).

4 Verstraeten S, Aimo L, Oteiza P. Aluminium and lead: molecular mechanisms of brain toxicity. Arch. Toxicol. 82, 789-802 (2008). 
5 Ventola CL. The antibiotic resistance crisis: part 1: causes and threats. $P$ T 40, 277-283 (2015).

6 Gao L, Giglio KM, Nelson JL, Sondermann H, Travis AJ. Ferromagnetic nanoparticles with peroxidase-like activity enhance the cleavage of biological macromolecules for biofilm elimination. Nanoscale 6, 2588-2593 (2014).

7 Wang $\mathrm{Y}$, Wang $\mathrm{K}$, Zhao J et al. Multifunctional mesoporous silica-coated graphene nanosheet used for chemo-

photothermal synergistic targeted therapy of glioma. J. Am. Chem. Soc. 135, 4799-4804 (2013).

8 Wallingford JB. Planar cell polarity and the developmental control of cell behavior in vertebrate embryos. Annu. Rev. Cell Dev. Biol. 28, 627-653 (2012).

9 Juriloff DM, Harris MJ. A consideration of the evidence that genetic defects in planar cell polarity contribute to the etiology of human neural tube defects. Birth Defects Res. A: Clin. Mol. Teratol. 94, 824-840 (2012).

10 Gandalovicova A, Vomastek T, Rosel D, Brabek J. Cell polarity signaling in the plasticity of cancer cell invasiveness. Oncotarget 7, 25022-25049 (2016).

11 Shelly M, Poo MM. Role of LKB1-SAD/MARK pathway in neuronal polarization. Dev. Neurobiol. 71, 508-527 (2011).

12 Malatesta M, Grecchi S, Chiesa E, Cisterna B, Costanzo M, Zancanaro C. Internalized chitosan nanoparticles persist for long time in cultured cells. Eur. J. Histochem. 59, 2492 (2015).

13 Barnes AP, Lilley BN, Pan YA et al. LKB1 and SAD kinases define a pathway required for the polarization of cortical neurons. Cell 129, 549-563 (2007). 